\title{
Frontières
}

\section{L'enfance et la mort : est-ce un paradoxe ?}

\section{Marie-Ange Abras}

Volume 13, numéro 1, automne 2000

La mort au tableau noir

URI : https://id.erudit.org/iderudit/1074251ar

DOI : https://doi.org/10.7202/1074251ar

Aller au sommaire du numéro

\section{Éditeur(s)}

Université du Québec à Montréal

ISSN

1180-3479 (imprimé)

1916-0976 (numérique)

Découvrir la revue

Citer ce document

Abras, M.-A. (2000). L'enfance et la mort : est-ce un paradoxe ? Frontières, 13(1), 62-64. https://doi.org/10.7202/1074251ar

\section{Résumé de l'article}

Des psychiatres ont constaté que les enfants s'éveillaient de plus en plus tôt aux notions de la sexualité et de la mort. Cependant, certains adultes pensent qu'il est paradoxal de parler de la mort avec des enfants qui commencent à s'ouvrir à la vie. Il sera tôt ou tard nécessaire de parler de la mort, et $a$ fortiori de la vie, avec les enfants. Même si le thème de la mort fait partie des enseignements reçus à l'école à l'occasion des cours de français ou de sciences biologiques, il est rarement le sujet d'une réflexion approfondie. Les occasions d'approfondir le thème de la mort sont pourtant multiples, que ce soit en observant un animal mort ou en lisant un conte, ce qui permet aux enfants de s'impliquer globalement. Face à la mort et à la perte, l'important est de laisser les enfants exprimer leurs émotions par rapport au deuil et à la souffrance. Il est également nécessaire de répondre à leurs questions (et même de les stimuler), quitte à avouer parfois qu'on ne sait pas.
Ce document est protégé par la loi sur le droit d'auteur. L'utilisation des services d’Érudit (y compris la reproduction) est assujettie à sa politique d'utilisation que vous pouvez consulter en ligne.

https://apropos.erudit.org/fr/usagers/politique-dutilisation/ 


$\begin{array}{llllllllll}P & O & \text { I } & N & T & D & E & V & U & E\end{array}$

\section{Résumé}

Des psychiatres ont constaté que les enfants s'éveillaient de plus en plus tôt aux notions de la sexualité et de la mort. Cependant, certains adultes pensent qu'il est paradoxal de parler de la mort avec des enfants qui commencent à s'ouvrir à la vie. Il sera tôt ou tard nécessaire de parler de la mort, et a fortiori de la vie, avec les enfants. Même si le thème de la mort fait partie des enseignements reçus à l'école à l'occasion des cours de français ou de sciences biologiques, il est rarement le sujet d'une réflexion approfondie. Les occasions d'approfondir le thème de la mort sont pourtant multiples, que ce soit en observant un animal mort ou en lisant un conte, ce qui permet aux enfants de s'impliquer globalement. Face à la mort et à la perte, l'important est de laisser les enfants exprimer leurs émotions par rapport au deuil et à la souffrance. II est également nécessaire de répondre à leurs questions (et même de les stimuler), quitte à avouer parfois qu'on ne sait pas.

Mots clés : enfance - deuil - temporalité

\section{Abstract}

Psychiatrists state that children are becoming aware of universal notions such as sex and death at an increasingly early age. Yet, some adults feel that it is a contradiction to talk about death with children, since they are symbolic of life. It is precisely because they are full of life that they need to be introduced to the topic of death. Talking with children about death - and obviously about life as well - will become necessary at some point. Although to a rudimentary degree, death is part of the school curriculum, as in paleontology or literature, it is rarely given much reflection. There are many opportunities to explore death, whether it is the discovery of a dead animal or when reading a story, at a time when children are in rapt attention. When faced with death and bereavement, children should be allowed to express their grief. Their questions should always be answered or if timid, they should be encouraged to ask, even though we may have to admit at times, that we do not know the answer.

Key words : childhood - mourning temporality

\section{L'enfance et la mort : est-ce un paradoxe?}

\author{
Marie-Ange Abras, \\ chercheure associée au laboratoire CRISE, \\ (Centre de Recherche sur I'Imaginaire Social et \\ I'Éducation) de I'Université Paris VIII et infirmière \\ pédagogue en soins palliatifs.
}

Françoise Dolto souligne que l'expérience de la naissance est la première émergence de l'expérience de la mort ${ }^{1}$. La psychanalyste française rappelle que nul n'a assisté à l'événement de sa naissance, mais l'expérience qui y est vécue marque le passage de l'état de fœtus à l'état d'enfant. D'un point de vue symbolique, la vie rythme le perpétuel enchaînement des transformations de soi pouvant être compris comme un processus de mort/renaissance. Il s'agit chaque fois d'un "mourir à soi » afin de « naître à soi » différemment.

La mort est donc inscrite au cœur de la vie. "Nous vivons de morts et nous mourrons de vie ", comme le disait joliment Héraclite. Or, dans la société moderne, la mort est tabou. Ce tabou est encore plus fort lorsqu'il s'agit d'enfants, car les adultes pensent qu'il faut les protéger de la souffrance. À côté de cette attitude de censure sur la mort, LouisVincent Thomas a observé un renouveau d'intérêt à l'égard de la mort, dû principalement au développement des soins palliatifs, aux suicides collectifs des sectes, au sida et au millénarisme. Pour Michel Hanus, ce retour de l'intérêt pour la mort concerne davantage le deuil et l'accompagnement des endeuillés que de la mort elle-même ${ }^{2}$. Toutefois, cet intérêt pour la mort n'a encore suscité que peu de recherches, dans le monde francophone, sur l'éducation à la mort dans les classes du primaire. Il semble qu'on veuille encore protéger les enfants contre la mort. On a constaté pourtant que l'éveil de l'enfant se fait de plus en plus tôt par rapport à des notions universelles comme la mort, la vie, l'amour et la sexualité ${ }^{3}$. On ne peut certes pas sous-estimer ces sentiments qui doivent être conceptualisés et verbalisés.

Dans les faits, l'enfant se retrouve souvent freiné dans ses interrogations sur la mort. Trop d'adultes ne se sentent pas le courage de parler de la mort et de la souffrance avec eux. Ils ont peur de les blesser, ou même de les sortir d'une sorte de bulle d'innocence qui les protégerait. Ils ne peuvent imaginer un seul instant qu'elle puisse être éducative. Cependant, contrairement aux adultes, 
les enfants ne séparent pas la mort de la vie. Pour un enfant, la mort n'est pas quelque chose de choquant, de rébarbatif ou d'horrible. Leurs sentiments sur la mort seront bien sûr éduqués par les parents. Il est certain que l'école peut contribuer à cette éducation.

\section{PARLER DE LA MORT À L'ÉCOLE}

Nous pouvons parler de la mort et de la vie, car "l'éducation peut tout " et elle "fait ce que nous sommes ", disait le philosophe français Claude Helvetius. Éduquer à la mort prend le sens d'une activité d'éveil à la fois philosophique, spirituelle, sociale et anthropologique. Il serait trop simple de présenter la mort comme un processus strictement biologique, comme il est réducteur de discuter de la sexualité sans aborder les questions existentielles et sociales. Selon Tony Anatrella, pour que les parents et les enseignants deviennent des éducateurs, c'est-à-dire des médiateurs entre l'enfant et la société, et des transmetteurs de valeurs, de savoir-faire, de savoir-être, il leur faut avoir une philosophie de l'existence. Il semble donc nécessaire d'aborder la mort avec les enfants de manière à en présenter la complexité et la richesse. On pourra notamment en parler à travers des activités qui impliquent l'art, la santé, la philosophie, les sciences humaines et les sciences biologiques.

La philosophie, par tradition, porte sa réflexion sur les problèmes éternels que sont le sens de la vie, de la mort, du temps, du destin, de l'origine et de la fin. On peut certes partir d'une activité philosophique, dans une classe du primaire, pour éduquer à la mort. Une ancienne sagesse philosophique, proposée par Socrate, puis reprise notamment par Montaigne, stipule que les arts de la vie impliquent l'acceptation de la mort. La vie ne pouvant être dissociée de la mort, on apprend à vivre en apprenant à mourir. On peut s'inspirer de cette conception de la sagesse pour proposer un programme d'éducation à la mort et au deuil dans les écoles. Il s'agira certes d'une éducation qui concerne la formation existentielle de l'enfant, une formation visant, comme le proposait encore Socrate, à sortir de l'ignorance de soi. Elle est foncièrement liée à l'acceptation de la mort comme un élément essentiel à la vie. Cette formation existentielle donne également l'occasion de réaliser que non seulement les concepts de vie et de mort se complètent en formant un tout, mais aussi qu'ils s'éclairent mutuellement.

\section{MORT ET TEMPORALITÉ}

La mort, comme concept et comme sentiment, est indéniablement associée à la durée de la vie. Par crainte de mourir, certains individus vont tenter de reporter ce moment ultime. Il arrive régulièrement que des individus vivent dans la peur de mourir ; pour fuir le moment présent, ils se projettent alors dans le futur ou rêvent d'un retour dans le passé. Il semble que l'acceptation du sentiment de sa propre mort renvoie à l'acception tragique du temps qui passe, qui s'écoule dans un éternel présent. La mort, en fait, ne peut survenir que dans le moment présent d'une vie. La fuite dans le passé ou dans le futur, par conséquent, est une fuite de la mort. Mais quel homme peut vivre avec le sentiment immédiat de sa mort ? Pour construire des empires, comme pour construire une famille, ne faut-il pas se projeter dans un avenir comme si la mort n'existait pas ? La temporalité, dans l'étendue de ses horizons infinis, assure ainsi une stabili-

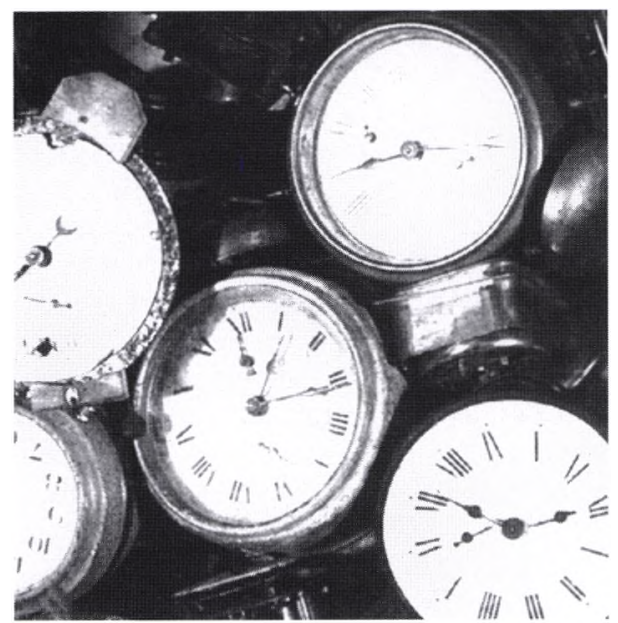

té émotive à l'homme qui cherche à construire son monde. Les hommes fuient la mort tout en sachant qu'ils ne pourront y échapper.

Qu'en est-il du concept de temps pour l'enfant ? On sait que le temps, pour un jeune enfant, n'a pas un sens de durée. Le temps pour lui est circulaire, cyclique, un peu comme le temps mythique des Anciens. Son monde se situe dans un présent perpétuel. Il vit au présent, sans avoir peur de mourir, sans mettre de distance envers la mort et les morts. Il va apprendre le temps chronologique, la durée, en se souvenant des secondes, des minutes, des heures, des jours et des années qui marquent les repaires de son existence : son âge, l'heure de la classe, l'heure de son programme télévisé, l'heure d'aller au lit, l'heure de se lever, etc. Ce temps chronologique qui organise l'existence, qui en radicalise l'ordonnancement, vient se superposer au temps cyclique de l'enfance. Le temps cyclique ne disparaît pas, il est enseveli sous le temps chronologique.

Peu à peu les enfants en viennent à obéir à une organisation chronologique du temps. L'institution scolaire, notamment, impose aux enfants une périodisation du temps qui les expulse de leur monde intemporel. Pourtant, le temps cyclique, qu'on qualifie souvent de " hors temps", est celui du mythe, du récit, de la fable et du conte. C'est un temps qui évoque l'infini, les possibles de l'imaginaire, la création. Dans la classe, tantôt l'enseignant insiste sur le temps chronologique pour éduquer à la discipline; tantôt il insiste sur le temps cyclique pour éduquer à la créativité. Lorsque l'enseignant sait rythmer ces deux temps de l'existence, il allie symboliquement le besoin d'ordre au besoin de désordre, le besoin de lois au besoin de transgression des lois, le besoin de vie et le besoin de mort.

Il semble important de permettre aux enfants de revenir au temps cyclique lors d'activités de lecture, de récit, de création. C'est dans ce temps cyclique que l'imaginaire crée ses plus beaux monstres et ses plus belles princesses vivant dans un temps et un espace hors d'atteinte de la réalité. Ce monde de l'imaginaire est celui qui exprime le mieux les sentiments et les désirs concernant la mort et la souffrance.

\section{LA PEUR ET LA MORT}

Selon Jiddu Krishnamurti, il existe deux sortes de peur : la peur physique (liée aux réflexes) et la peur psychologique. L'animal ne pense pas la peur et agit pour s'éloigner d'un danger potentiel. L'animal a une réaction spontanée aux stimuli de son milieu ${ }^{4}$. Quant à l'homme, il peut faire le constat de ses réactions, notamment celle de la peur. Il sait que sa peur peut devenir un véritable handicap (inhibition) qui l'empêche de répondre efficacement à une situation donnée. Outre le système nerveux central, l'être humain est muni de deux systèmes nerveux : "les systèmes sympathique et parasympathique ${ }^{5}$ ". Schématiquement, le système sympathique provoque chez l'adulte, quelle que soit sa réaction face à la peur, une incapacité à courir, des mains qui suent, une bouche sèche et une contraction musculaire. Le système parasympathique réagit en fonction du principe de plaisir entraînant une salivation, des yeux brillants et la sensation de " bon- 
heur ". Dans le quotidien, l'homme cherche à contrôler la fonction du système sympathique et à perpétuer l'action $\mathrm{du}$ système parasympathique (il recherche le plaisir et fuit la peur). Pourtant la fonction du sympathique et du parasympathique reste un mécanisme physiologique ; aussi longtemps que l'homme s'identifie à celui-ci, il n'apprend pas véritablement à surmonter sa peur.

L'enfant ignore ce qui est dangereux et désire en faire l'expérience. Certains enfants peuvent avoir envie notamment de toucher le feu, et il est possible qu'ils se brûlent alors le bout des doigts. Il est évident que si un enfant se brûle, il aura très vite retiré sa main du feu, ne pouvant supporter la douleur. La réaction de retirer sa main du feu a été spontanée. Cette expérience ne va pas nécessairement provoquer un sentiment de peur du feu. D'où vient le sentiment de peur? On constate, entre autres, que la peur relève d'un apprentissage. Elle peut provenir notamment de certaines réflexions des parents comme : "si tu ne fais pas ce que je t'ai demandé, tu seras puni " ou "si tu parles de la mort à ton âge, tu perdras l'envie de vivre ". L'enfant va donc réagir en faisant plaisir à ses parents et par le fait même, il va intérioriser le sentiment de peur. Il s'agit d'un comportement appris. L'adulte fuit la peur qui fait de son existence un champ de batailles et de contradictions. Il projette et transmet à l'enfant ses idées, ses concepts et ses sentiments sur la mort. Il forme ainsi le sentiment de l'enfant sur la mort. Il l'éduque, d'une certaine manière, à réagir avec un sentiment de peur, si tel est le cas, devant la mort. En fait, lorsque les enfants expriment leur peur face à la mort, ils ne font souvent que répéter ce qu'ils ont appris des parents.

Il est préférable que les enfants puissent exprimer leurs émotions sur la vie et la mort. Élisabeth Kübler-Ross souligne le conditionnement néfaste des enfants conduisant à un manque d'initiative, d'autonomie affective et de confiance en soi ; tous des manques pouvant mener à l'aigreur, à l'insatisfaction, à l'asocialité, à la récrimination constante et finalement à la fragilisation face à la maladie. Les enfants ont besoin de vivre leurs émotions, car pour eux, il n'y a pas a priori de bonnes ou de mauvaises émotions. Pour les parents, la peur de la mort est une émotion ambiguë ; ils cherchent à se protéger de la crainte et des questions qu'elle suscite : " Le problème dans notre société, c'est aussi que, pour les enfants, la mort n'a plus de réalité ; elle est évacuée, notamment parce qu'on ne les emmène plus aux enterrements $» 6$.

Les enfants, spontanément, jouent avec leurs émotions. Le jeu est un moyen actif de se protéger et d'exprimer en même temps des émotions très fortes. Ils matérialisent leur peur de la mort sur un objet ou sur une histoire, lui donnant une forme (phénomène de la catharsis des anciens) afin de s'en délivrer. Mais surtout " ce que les enfants cherchent tout le temps, c'est l'inconnu qu'ils ne connaissent pas encore, et c'est pour ça qu'ils jouent avec la mort, et qu'il faut les laisser jouer avec la mort $^{7} "$. Les enfants jouent à être morts et à donner la mort, "justement parce qu'ils sont dans la vie, et ils ont tout à fait raison parce que c'est en donnant la mort imaginaire que la vie, dans sa réalité, prend tout son sens, toute sa verdeur, seulement c'est une mort imaginaire ${ }^{8} »$. Le jeu est donc une réalité qui renvoie au temps cyclique. Selon Dolto, les adultes également aiment jouer ou se donner la mort dans leurs rêves : "Ce sont nos pulsions de vie qui font ça, comme les enfants; l'inconscient ne veut pas pour autant mourir, au contraire, c'est pour mieux vivre qu'il donne la mort, c'est pour jouer ${ }^{9}$ ». En somme, le jeu, dans la réalité de l'enfant ou dans le rêve de l'adulte, vise la maîtrise symbolique des émotions. Il faut encourager le jeu dans la classe, autour de saynètes sur le thème de la vie et de la mort, parce qu'il permet l'expression d'émotions fortes qui trouvent difficilement à se dire autrement.

\section{CONCLUSION}

L'éducation de l'enfant à la mort demande souvent de la part des adultes une remise en question de leurs croyances, de leurs valeurs et de leurs expériences existentielles. Communément, nous pensons qu'il est contraire à la vie que l'enfant puisse parler de la mort, alors que les expériences de la mort font partie de son existence. C'est pourquoi il est nécessaire de répondre aux questions des enfants à ce sujet, et même de les stimuler. On peut aborder le thème de la mort, dans la classe, de différentes manières : par le récit d'une histoire, par le jeu de rôle, par un questionnement philosophique, par la lecture d'un poème ou par l'observation d'un animal décédé dans le laboratoire de biologie. En fait, les occasions sont nombreuses d'approfondir la réflexion sur la mort. On sait que de laisser les enfants s'exprimer sur la mort ne les perturbe pas, au contraire, nous pensons que cela aide à leur développement.

\section{Notes}

1 Françoise DOLTO, Parler de la mort, Paris, Éditions Gallimard, Collection Le petit mercure, 1998, p. 10.

2 Michel HANUS et Barbara $M$. SOURKES, Les enfants en deuil : portraits du chagrin, Paris, Éditions FrisonRoche, 1997.

3 Ginette RAIMBAULT, L'enfant et la mort : problèmes de la clinique du deuil, Paris, Dunod, 1995 (1975).

4 La réaction instinctive est provoquée par un stimulus donné aux surrénales par le système nerveux sympathique.

5 Sarah BRASH, Gina MARANTO, Wendy MURPHY et Bryce WALKER, Le cerveau : l'aventure technologique, Paris, Éditions France-Loisirs, 1992, p. 26.

6 Christiane OLIVIER, L'ogre intérieur, Paris, Fayard, 1999.

7 DOLTO, ibid. note 1, p. 47.

8 Ibid., p. 45.

9 Ibid., p. 46. 